\title{
A Wavelet-Based Method for the Determination of the Relative Resolution Between Remotely Sensed Images
}

\author{
Jorge Núñez, Octavi Fors, Xavier Otazu, Vicenç Palà, Román Arbiol, and Maria Teresa Merino
}

\begin{abstract}
Spatial resolution is a key parameter of all remote sensing satellites and platforms. The nominal spatial resolution of satellites is a well-known characteristic because it is directly related to the area in ground that represents a pixel in the detector. Nevertheless, in practice, the actual resolution of a specific image obtained from a satellite is difficult to know precisely because it depends on many other factors such as atmospheric conditions. However, if one has two or more images of the same region, it is possible to compare their relative resolutions. In this paper, a wavelet-decomposition-based method for the determination of the relative resolution between two remotely sensed images of the same area is proposed. The method can be applied to panchromatic, multispectral, and mixed (one panchromatic and one multispectral) images. As an example, the method was applied to compute the relative resolution between SPOT-3, Landsat-5, and Landsat-7 panchromatic and multispectral images taken under similar as well as under very different conditions. On the other hand, if the true absolute resolution of one of the images of the pair is known, the resolution of the other can be computed. Thus, in the last part of this paper, a spatial calibrator that is designed and constructed to help compute the absolute resolution of a single remotely sensed image is described, and an example of its use is presented.
\end{abstract}

Index Terms-Image resolution, relative resolution, wavelet decomposition.

\section{INTRODUCTION}

$\mathbf{T}$ HE SPATIAL resolution of remote sensing satellites and platforms, besides the spectral, radiometric, and temporal resolution, is a key parameter for all scientific and commercial purposes. The nominal spatial resolution of remote sensing satellites has been in constant improvement during the last

Manuscript received April 15, 2005; revised February 18, 2006. This work was supported in part by the Ministerio de Ciencia y Tecnología (MCyT) of Spain under Grants AyA2001-3092, AyA2005-08604, and TIN2004-02970, and in part by the Institut Cartogràfic de Catalunya, Generalitat de Catalunya. The work of X. Otazu was supported by the program Ramón y Cajal supported by the Spanish Ministry of Science and Technology. The work of M. T. Merino was supported by a research fellowship (FPU) from the Spanish Ministry of Education and Science.

J. Núñez and O. Fors are with the Departament d'Astronomia i Meteorologia, Universitat de Barcelona, E-08028 Barcelona, Spain, and also with the Observatori Fabra, E-08028 Barcelona, Spain (e-mail: jorge@am.ub.es; ofors@ am.ub.es).

X. Otazu is with the Centre de Visió per Computador, Universitat Autónoma de Barcelona, E-08193 Bellaterra, Spain (e-mail: xotazu@cvc.uab.es).

V. Palà and R. Arbiol are with the Institut Cartogràtic de Catalunya, Parc de Montjuïc, E-08038 Barcelona, Spain (e-mail: vicencp@icc.es; arbiol@icc.es).

M. T. Merino is with the Departament d'Astronomia i Meteorologia, Universitat de Barcelona, E-08028 Barcelona, Spain (e-mail: mmerino@am.ub.es).

Digital Object Identifier 10.1109/TGRS.2006.875359
TABLE I

Nominal Spatial Resolution of SOME REMOTE SENSING SATELLITES

\begin{tabular}{lcc}
\hline & Resolution (m) & \\
\cline { 2 - 3 } Satellite & Multispectral & Panchromatic \\
\hline LANDSAT -7 & 30.0 & 15.0 \\
SPOT -4 & 20.0 & 10.0 \\
SPOT -5 & 5.0 & 2.5 \\
IKONOS & 4.0 & 1.0 \\
QuickBird & 2.5 & 0.6 \\
\hline
\end{tabular}

years. Table I shows the nominal spatial resolution of some of these satellites.

It is well known that an analog optical system is fully characterized by its point spread function (PSF) and, consequently, by its Fourier transform known as the optical transfer function (OTF). For resolution aspects, we consider the modulation transfer function (MTF), which is the modulus of the OTF. In digital imaging, analog images produced by the optical system are sampled by the detector. If the images are properly oversampled (with a frequency higher than the Nyquist frequency), the resolution properties are preserved. However, due to design constraints, this is not the real case, and most imaging sensors (including remote sensing satellites such as Landsat and SPOT) produce digital images by undersampling the analog input images with the consequence that the resulting resolution is fixed mainly by spatial sampling and secondarily by the properties of the MTF. Hence, in satellite-based platforms, the nominal resolution is directly related to the area in ground that represents a pixel in the detector. Thus, it is related to the distance from satellite to ground (satellite orbit), pixel size, and focal length of the observing camera or instrument.

On the other hand, in practice, the true resolution of an image is also related to the size of the PSF of the whole observing system, which includes not only the optics and the detector but also the atmospheric conditions, the illumination of the scene, and many other factors. The atmospheric conditions also depend on the turbulence, the quantity of water vapor of the atmosphere, the atmospheric scattering caused by the dust in suspension, aerosol concentration, air pollution, and other factors [1]-[4]. All these factors are difficult or impossible to determine with enough accuracy at any time.

In particular, for some authors [3], [4], the atmospheric blur caused by scattering can significantly affect the spatial resolution in remote sensing observations. Thus, the actual practical spatial resolution of a particular remotely sensed image is difficult to calculate accurately. One method to overcome the problem is to know precisely the size of several features appearing on the image so we can calibrate the absolute spatial resolution. 
However, if we have more than one image of the same region, which is obtained by either the same or different platforms, it is possible to compare the relative resolution between both images. If we know the absolute resolution of one of them, we could compute the resolution of the other. Otherwise, we will have at least a comparison between the quality of both images and its relative resolution. This could be very important in the comparison of the actual resolution between two different satellite-based platforms.

Another possible application could be to estimate the degree of increment of the resolution obtained by a superresolution method [5]. By computing the relative resolution between the superresolution result and the images contributing to such a result, we could estimate the increment of resolution (superresolution) achieved.

Thus, in an effort to solve the problem of determining the relative resolution between images, in the first part of this paper, we propose a wavelet-decomposition-based method for the determination of the relative resolution between two remotely sensed images of the same area. Furthermore, in Section IV, we describe the form and use of a spatial calibrator that we have constructed to help compute the absolute resolution of a single remotely sensed image.

Although, in principle, the method proposed in this paper could be applied to both electronic imaging and digitized (scanned) photography, in practice, the latter introduces other parameters related to both the photographic process itself (i.e., type and density of the emulsion, color or black and white negative, sensitivity, preprocessing, and development) and the scanning process (i.e., type of scanner, physical or interpolated resolution, and accuracy of scanning). These factors, in addition to the nonlinearity of the photographic plate, make the comparison between two different images obtained from digitized photographies very difficult unless a complicated system of calibration is carried out. Thus, in this paper, we will only consider the relative resolution for the case of digital images from spatially based platforms.

\section{Algorithm for Estimating the Relative RESOLUTION BETWEEN TWO IMAGES}

\section{A. Method Outline}

The main idea in which the proposed method for evaluating the relative resolution between a pair of images is based consists of the following.

1) Preprocess the images by registering (if needed) the lowresolution image to the same size as the high-resolution one to be superimposed, and perform (if needed) histogram matching between both images.

2) Obtain a series of decreasing resolution versions of the higher resolution image.

3) Compare quantitatively these images with the lower resolution image of the original pair.

4) Obtain a point of maximum correlation between the images of the series and the low-resolution image of the pair. If we use the higher resolution image to obtain the series, and this series is long enough, the correlation should present a maximum.

5) From this maximum, compute the relative resolution between the images of the pair.

\section{B. Preprocessing the Images}

To carry out the registration, in this paper, the images are registered up to within 0.25 pixels root mean square (rms) by resampling the low-resolution image using control points and a bicubic polynomial fit. It is important to point out that, as in all other cases involving registration (for example, the image fusion), the better the registration, the better the method will work. However, because the proposed method is based on quantitative correlations between the low-resolution image of the pair and the images of a series of decreasing resolution versions of the higher resolution image, the maximum correlation will take place at the resolution level of the lower resolution one. Hence, registration is not a critical issue of the method (indeed, it is less critical than in an image merging process).

To take into account the spectral differences (if any), i.e., the different atmospheric and illumination conditions, between the two images of the pair, we performed a conventional histogram matching between them. Specifically, after computing the histogram of both images, the histogram of the low-resolution image is used as the reference to which we match the histogram of the high-resolution one. Indeed, if both images were obtained by the same platform under similar conditions, this first step could be skipped.

If one of the images of the pair is multispectral and the other is panchromatic, we can compare the relative resolution between the panchromatic image and each one of the spectral bands of the multispectral image. However, although these comparisons are possible (and already carried out in the examples hereinafter), given the very different spectral response functions (SRFs) of the panchromatic and multispectral sensors [6] and that the present method is based on correlations between images, the best way to compare the relative resolution between a multispectral image and a panchromatic image should be to use the intensity component of the multispectral one. This intensity component should be computed in a way that the result should be spectrally as close as possible to the panchromatic image.

If both images are multispectral, we can compute, in principle, the relative resolution between any pair of spectral bands, but because of the considerations exposed above, again, the best way would be either to compare the resolution between bands of similar SRF or to compute the intensity component of both images as before. In the examples in this paper, we used the intensity component $L$ of the multispectral images using the expression $L=(R+G+B) / 3$ with preference to other expressions as $I=\max (R, G, B)$ or $L^{\prime}=(\max (R, G, B)+\min (R, G, B)) / 2$. This choice was due to the spectral characteristics of the images used (SPOT-3, Landsat-5, and Landsat-7).

\section{Wavelet Decomposition}

To obtain the series of successive lower resolution versions of the higher resolution image of the pair, we can use several techniques as, for example, filtering iteratively the image with a low-pass filter, e.g., by convolving the image successively with a Gaussian function of a known standard deviation. However, in this paper, we preferred to use the well-established theory of wavelet decomposition [7]-[12]. This is because using the wavelet decomposition we can mathematically know the 
resolution of each image of the obtained series with respect to the original image.

Multiresolution analysis based on wavelet decomposition is increasingly being used for the processing of images [13]-[16], particularly for the analysis of images in remote sensing [17], and extensively used in the field of image merging [6], [18]-[23].

The advantage of wavelet-based multiresolution analysis for our application is that the wavelet transform provides a framework to decompose images into a number of new images, each one of them with a different degree of resolution. This allows the construction of a series of images of decreasing resolution.

To obtain a shift-invariant discrete wavelet decomposition of an image, we follow Starck and Murtagh [24] and use the discrete wavelet transform known as "à trous" ("with holes") algorithm [25] to decompose the image into wavelet planes (see, for example, [21] for details).

The "à trous" method (see [26] and [27]) consists of a shift-invariant symmetric dyadic oversampled (undecimated) discrete wavelet transform. In practice, it leads to a bandpass stack of images with the same dimensions (i.e., no decimation, thus no pyramid) with a reduction of the resolution by a factor of 2 from level to level using a Gaussian-like low-pass filter. With regard to the filter, we used in this paper the standard "à trous" filter, which is based on a scaling function with a $\mathbf{B}_{3}$ spline profile. The scaling function with a $\mathbf{B}_{3}$ spline profile is compact in both direct and Fourier spaces, is smooth, symmetric, and converges rapidly to zero. This filter fully satisfies the conditions and constraints imposed by Shensa [26] for a "à trous" filter. There are also other possible filters, such as the "à trous" alternative linear filter, but as stated above, we will prefer the standard filter to obtain a dyadic decomposition. Therefore, for all of the above, we consider the combination of the "à trous" algorithm and the standard low-pass filter (based on a scaling function with a $\mathbf{B}_{3}$ spline profile) as the best suited for our study.

In the practical implementation, given an image $\mathbf{p}_{0}$, we use the "à trous" algorithm to construct the sequence of approximating images $\mathbf{p}_{l}(l=1, \ldots, n)$, which are versions of the original image $\mathbf{p}_{0}$ at increasing scales (i.e., decreasing resolution levels). Because in this representation we are using a dyadic decomposition scheme, i.e., the original image $\mathbf{p}_{0}$ has twice the resolution of $\mathbf{p}_{1}$ and the image $\mathbf{p}_{1}$ has a double resolution than $\mathbf{p}_{2}$. If the resolution of image $\mathbf{p}_{0}$, for example, is $10 \mathrm{~m}$, the resolution of $\mathbf{p}_{1}$ would be $20 \mathrm{~m}$, and the resolution of $\mathbf{p}_{2}$ would be $40 \mathrm{~m}$.

Note, however, that, as stated above, in this process, all the consecutive approximating images have the same number of pixels as the original image. This is a consequence of the fact that the "à trous" algorithm is a nonorthogonal oversampled (redundant) transform [12]. This is a restriction on the use of this particular wavelet approach for applications such as image compression.

\section{Image Correlation}

Once the series of successive lower resolution versions of the higher resolution image is obtained, we proceed to quantitatively compare these images with the lower resolution image of the original pair. In this paper, we performed it by computing

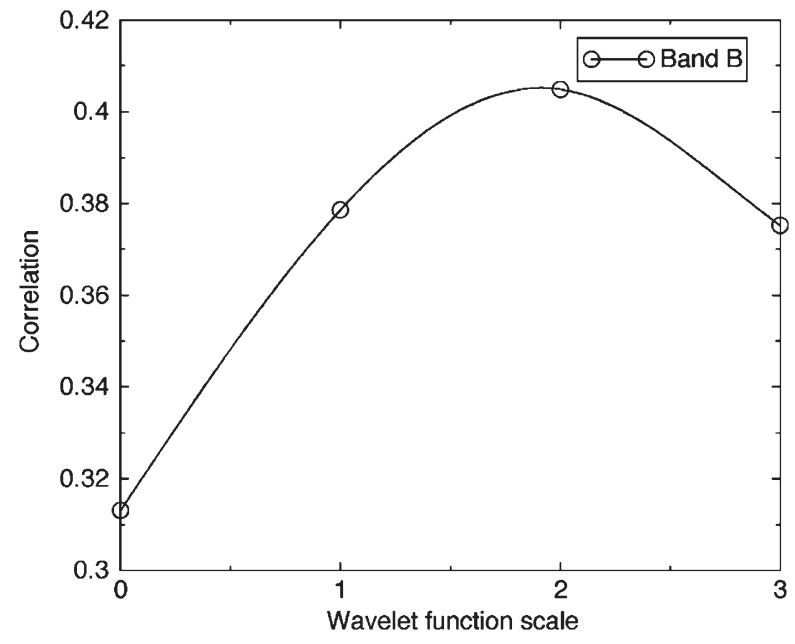

Fig. 1. Example of a correlation curve between the high- and low-resolution images as a function of the scale of the wavelet function.

the correlation coefficient between the images using the standard coefficient

$$
\operatorname{Corr}(A / B)=\frac{\sum_{j=1}^{n p i x}\left(A_{j}-\bar{A}\right)\left(B_{j}-\bar{B}\right)}{\sqrt{\sum_{j=1}^{n p i x}\left(A_{j}-\bar{A}\right)^{2} \sum_{j=1}^{n p i x}\left(B_{j}-\bar{B}\right)^{2}}}
$$

where $\bar{A}$ and $\bar{B}$ represent the mean value of the corresponding data set. For positive images, $-1<\operatorname{Corr}(A / B)<1$.

\section{E. Maximum of Correlation}

Using (1), we compute the correlation between each one of the images of the series and the lower resolution image. The result is a series of correlation numbers that, as stated above, if the series is long enough, should present a maximum. For example, if the resolutions of the high- and low-resolution images of the pair were 10 and $30 \mathrm{~m}$, respectively, the series of successive lower resolution versions of the first image would have resolutions of $10 \mathrm{~m}$ (scale 0 ), $20 \mathrm{~m}$ (scale 1), $40 \mathrm{~m}$ (scale 2), and $80 \mathrm{~m}$ (scale 3). Hence, the series of correlation numbers between such images and the 30-m image would present a maximum at some point between the 20- and 40-m images of the series.

To compute such maximum, in this paper, we fit a cubic spline function to the obtained series of correlation numbers as a function of the scale. Fig. 1 shows an example of a correlation curve obtained using real data coming from SPOT-3 (10-m nominal resolution) and Landsat-5 (30-m nominal resolution) satellites (see examples below). Note that Fig. 1 represents an extreme case of the application of the proposed method because, in it, we are comparing the panchromatic band of the SPOT-3 and the blue band of the Landsat-5 satellites, which have very different SRFs. Even so, it is easy to see that the maximum of the correlation curve is between scales 1 and 2, i.e., between 20 and $40 \mathrm{~m}$.

It is interesting to note here that the series of lower resolution versions of the higher resolution image could be obtained by other methods, such as, for example, by Gaussian convolution with a kernel of known full-width at half-maximum (FWHM). In this case, a curve similar in form to that in Fig. 1 would be obtained. Fig. 2 shows an example of such a curve as correlation versus FWHM of the Gaussian kernel. In Fig. 2, which 


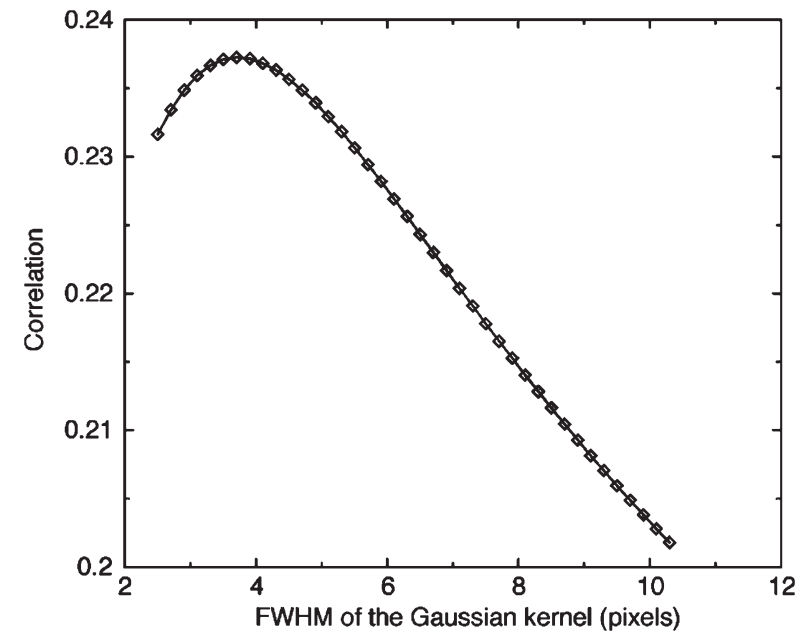

Fig. 2. Example of a correlation curve between the high- and low-resolution images as a function of the FWHM of the Gaussian filter.

corresponds to the comparison between the panchromatic band of the SPOT-3 satellite and the intensity component of the Landsat-5 satellite, the maximum correlation is reached at approximately FWHM = 3.70. However, as commented below, the relationship between the FWHM of the maximum of the curve and the resolution is not as straightforward to derive as in the case of wavelets.

\section{F. Relative Resolution}

As stated above, one of the advantages of using the wavelet approach is the dyadic decomposition scheme that allows an immediate correspondence between the scale of the decomposition and the resolution. This is a consequence of the properties of wavelets. Thus, the relationship between the scale of the wavelet decomposition and the relative resolution with regard to the first image of the series (scale 0 or original high-resolution image) is

$$
Y=2^{X}
$$

where $X$ is the scale and $Y$ is the relative resolution between the images of the pair.

Note that in the case in Fig. 2 (i.e., Gaussian convolution in place of wavelet decomposition), we do not have a dyadic scheme. So, we cannot use a direct expression such as (2) to easily compute the relative resolution from the FWHM of the maximum of the curve, and an accurate calibration would be needed. This is a consequence of the lack of orthogonal properties of this decomposition scheme.

It is important to point out here that because the proposed method is based on the correlation between the whole lowresolution image and the series of decreasing resolution images obtained from the high-resolution one, we are obtaining the relative resolution between the particular pair of images considered. These images include not only the size of the PSF (which is determined by the detector, the optics, and the atmospheric conditions) but also the particular spatial frequency contents of the observed scene. In other words, if we are observing, for example, using two different satellites of different nominal resolution, a featureless snowfield or the surface of the sea, the proposed method would give both images approximately the same resolution. However, if with the same instruments we observe a scene of rich spatial frequency contents like an urban area, the method will find that both images have different resolutions and will compute the ratio between them.

\section{EXAMPLES OF RELATIVE RESOLUTION}

\section{A. Simulated Data}

To check the robustness and accuracy of the method, we applied the preceding methodology to compute the relative resolution between pairs of different resolution-simulated images of which we know theoretically the relative resolution between them.

In the first example, we used as a high-resolution image the image in Fig. 5, which was obtained by the panchromatic camera of the Landsat-7 satellite. For the low-resolution images, we used the first, second, and third wavelet approximation images (i.e., $\mathbf{p}_{1}, \mathbf{p}_{2}$, and $\mathbf{p}_{3}$ ) of its own high-resolution image. As stated above, in a dyadic decomposition scheme, these approximation images have, respectively, two, four, and eight times lower resolution than the original image. Note that, in this example, we are using wavelet decomposition not only to obtain the series of decreasing resolution images of the high-resolution image of the pair but also to obtain the own low-resolution image of the pair. Hence, in this example, the differences between the expected and the obtained values for the relative resolution should come, almost exclusively, from the cubic spline used to fit each set of correlations.

In the second example, we used again the same highresolution image in Fig. 5, but, as lower resolution images, we synthetically generated three images of, respectively, 2, 2.5, and 3 times lower resolution. To obtain them, in the first step, we reduced the size of the high-resolution image using the appropriate resizing factor and, in the second step, expanded them again to the original size. We used bicubic interpolation in both steps. Following the above-described methodology, we used wavelet decomposition to obtain a series of lower resolution images of the high-resolution image for each pair, we used (1) to compute the correlation of each image of the series and the corresponding low-resolution image, and we fitted a cubic spline to each set of correlations. Figs. 3 and 4 show the obtained curves for the first and second examples, respectively.

As expected, all curves present a maximum from which we computed the relative resolution of each pair. In the first example, the maxima of the correlation are logically unity, whereas in the second example, the maxima are very close to unity but vary slightly (from 0.95 to 0.97 ) between the three simulated low-resolution images. This small variation is a normal consequence of the resizing and interpolation method used to generate them.

The abscissa of the maxima of the correlation curves of both examples, the relative resolution computed using (2), and the expected values are summarized in Table II. In the first example, the differences between the computed and expected relative resolutions are $2.0 \%, 0.5 \%$, and $0.0 \%$. As cited above, these errors are a consequence of the use of a cubic spline to fit the correlation sets. In the second example, these differences are $3.0 \%, 1.6 \%$, and $1.0 \%$. In all cases, the differences between 


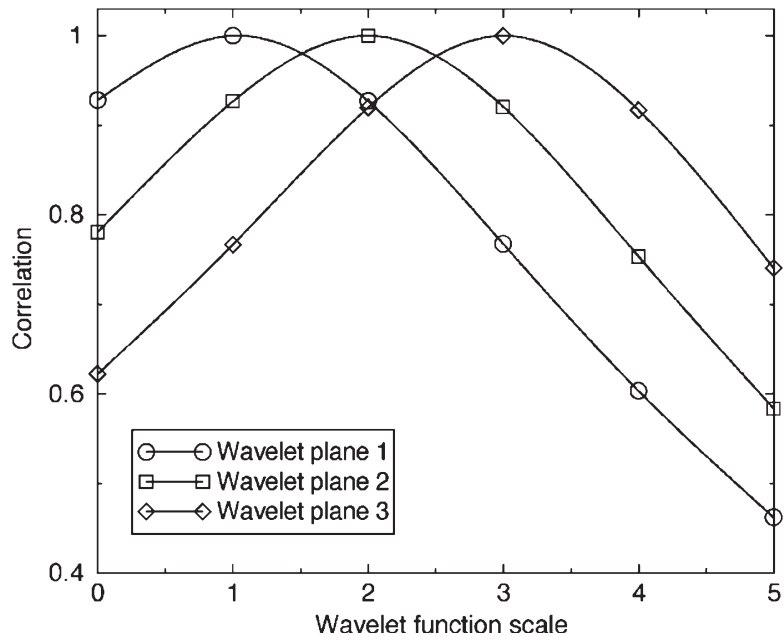

Fig. 3. Correlation curves between the high-resolution image displayed in Fig. 5 and its own first, second, and third approximation images (which have resolution that is two, four, and eight times lower, respectively) as a function of the scale of the wavelet function.

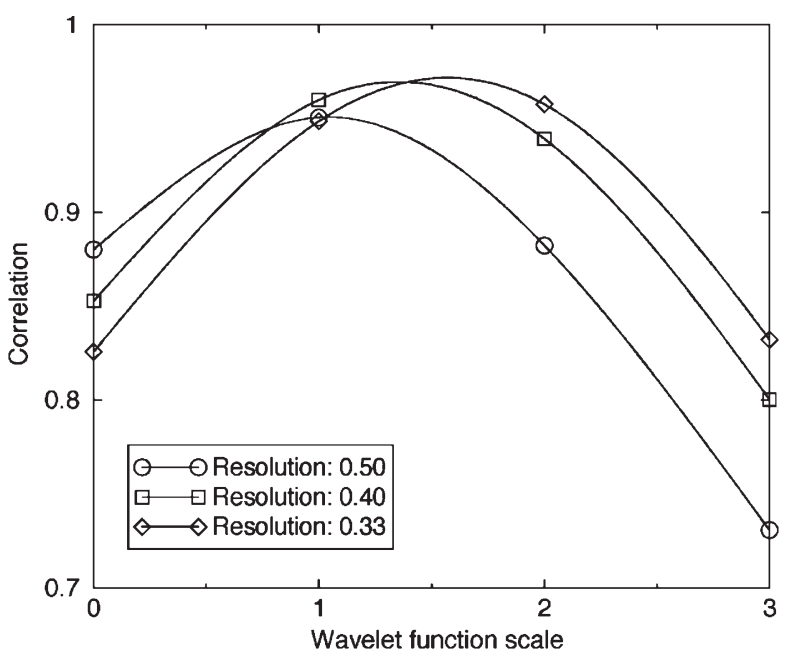

Fig. 4. Correlation curves between the high-resolution image displayed in Fig. 5 and three computer-generated images with resolution that is $2,2.5$, and 3 times lower, respectively, as a function of the scale of the wavelet function.

TABLE II

Relative Resolution Between Simulated IMAGES COMPUTED BY WAVELETS

\begin{tabular}{lccc}
\hline & $\begin{array}{c}\text { Maximum of } \\
\text { correlation }\end{array}$ & $\begin{array}{c}\text { Computed relative } \\
\text { resolution }\end{array}$ & $\begin{array}{c}\text { Expected } \\
\text { relative }\end{array}$ \\
Satellites & curve $(X)$ & $Y=2^{X}$ & resolution \\
\hline
\end{tabular}

High resol./

Approx. image

\begin{tabular}{llll}
$1 / 2$ resol. & 1.031 & 2.04 & 2 \\
$1 / 4$ resol. & 1.994 & 3.98 & 4 \\
$1 / 8$ resol. & 3.000 & 8.00 & 8 \\
\hline
\end{tabular}

High. resol/

Resized image

\begin{tabular}{lllc}
$1 / 2.0$ resol. & 1.041 & 2.06 & 2 \\
$1 / 2.5$ resol. & 1.345 & 2.54 & 2.5 \\
$1 / 3.0$ resol. & 1.570 & 2.97 & 3 \\
\hline
\end{tabular}

the computed and expected relative resolution are very small, which shows that the proposed method is robust and accurate enough to be applied to real data examples.

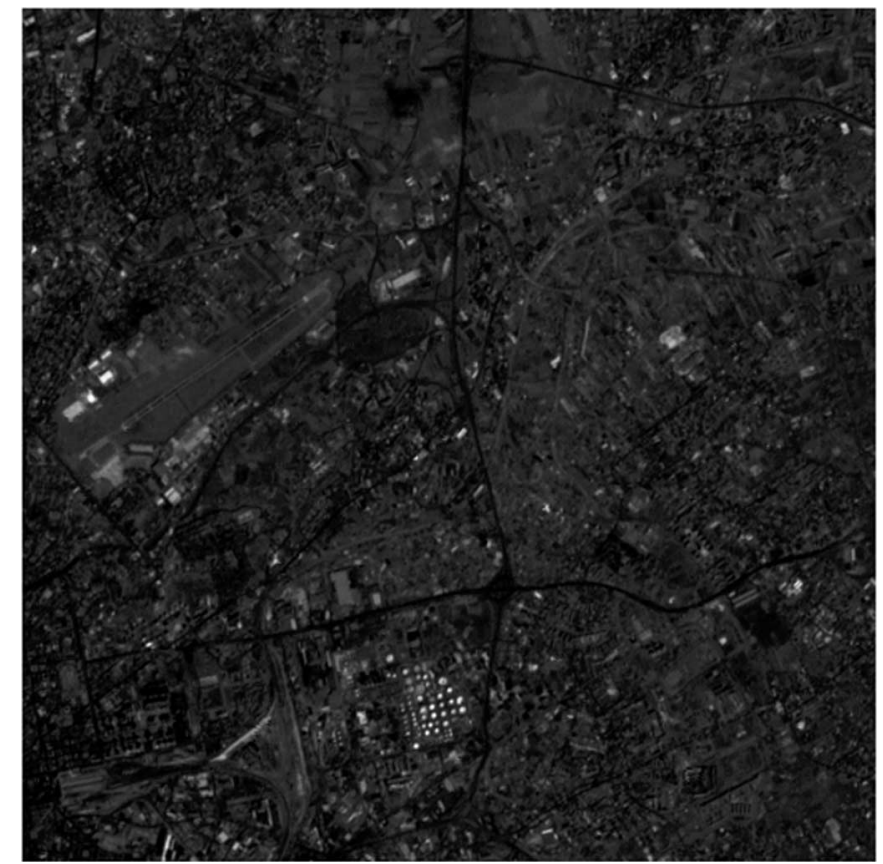

Fig. 5. High-resolution $(15 \mathrm{~m})$ panchromatic image of Naples obtained by the Landsat-7 satellite.

\section{B. Real Data}

As a first application to real data, we applied the preceding methodology to compute the relative resolution between two pairs of images obtained from different sensors.

The first pair of images was obtained by the same satellite (Landsat-7) during the same passage, but using two different instruments. They show a detail of the Italian city of Naples showing, thus, an urban area. Fig. 5 shows the high-resolution image of the pair. It was obtained using the panchromatic sensor of the Enhanced Thematic Mapper Plus (ETM+) of the satellite that has a nominal spatial resolution of $15 \mathrm{~m}$. The lowresolution image corresponds to the multispectral sensor of the $\mathrm{ETM}+$, which has a nominal spatial resolution of $30 \mathrm{~m}$. We used separately the spectral bands 1 (blue), 2 (green), and 3 (red) of the multispectral sensor, but, as stated above, to compare its resolution with respect to the panchromatic image in the best way, we also transformed this image to panchromatic considering only the intensity component. The intensity component was computed as the mean of the three channels (i.e., 1, 2, and 3 ) of the multispectral image. Fig. 6 shows the lowresolution image (i.e., $30 \mathrm{~m}$ ) once transformed to panchromatic. Indeed, both images of the pair belong to the same epoch of the year and present the same conditions of illumination.

To show a very different situation, the second pair of images presents two images of a nonurban area obtained from different satellites in different epochs of the year. They show detail of an Argentinean landscape, which includes several agricultural lots, roads, a river bed, and a small town. Fig. 7 shows the highresolution image of the pair, which is a $10-\mathrm{m}$ nominal resolution panchromatic image obtained by the SPOT-3 satellite. The lowresolution image of the pair was obtained with the multispectral sensor of the Landsat-5 satellite that has a nominal spatial resolution of $30 \mathrm{~m}$. In this case, we used as spectral bands the three (i.e., $R, G$, and $B$ ) pseudochannels computed using the usual transformation, i.e., $R=\left(B_{5}+B_{7}\right) / 2$, 


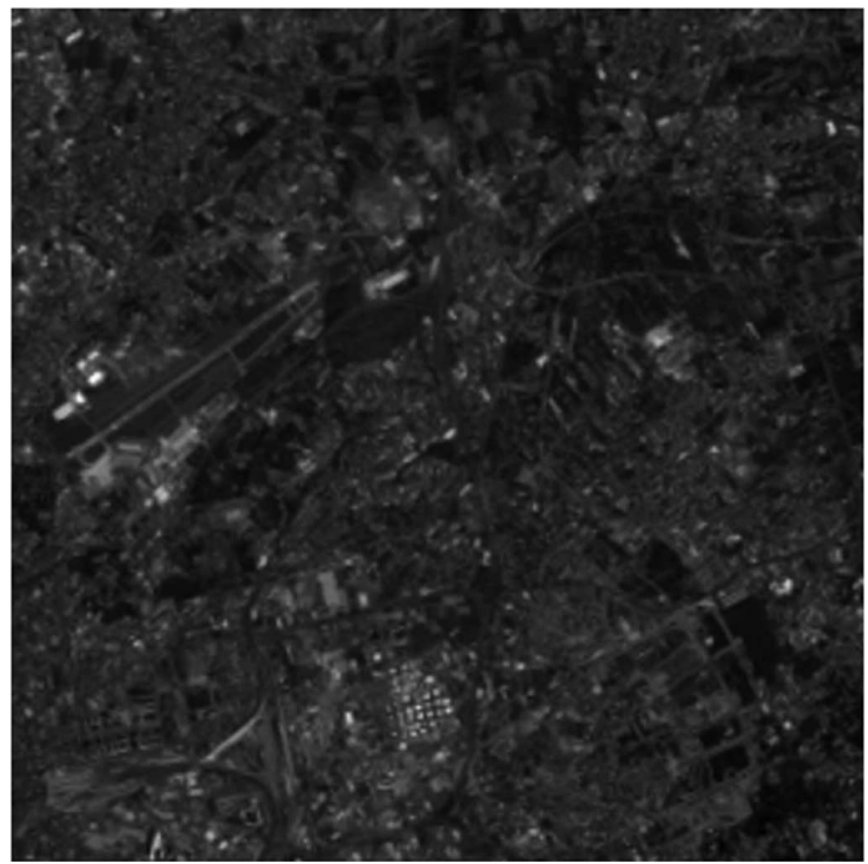

Fig. 6. Low-resolution $(30 \mathrm{~m})$ multispectral image of the same area as Fig. 5 obtained simultaneously by the Landsat- 7 satellite. Image was transformed to intensity levels.

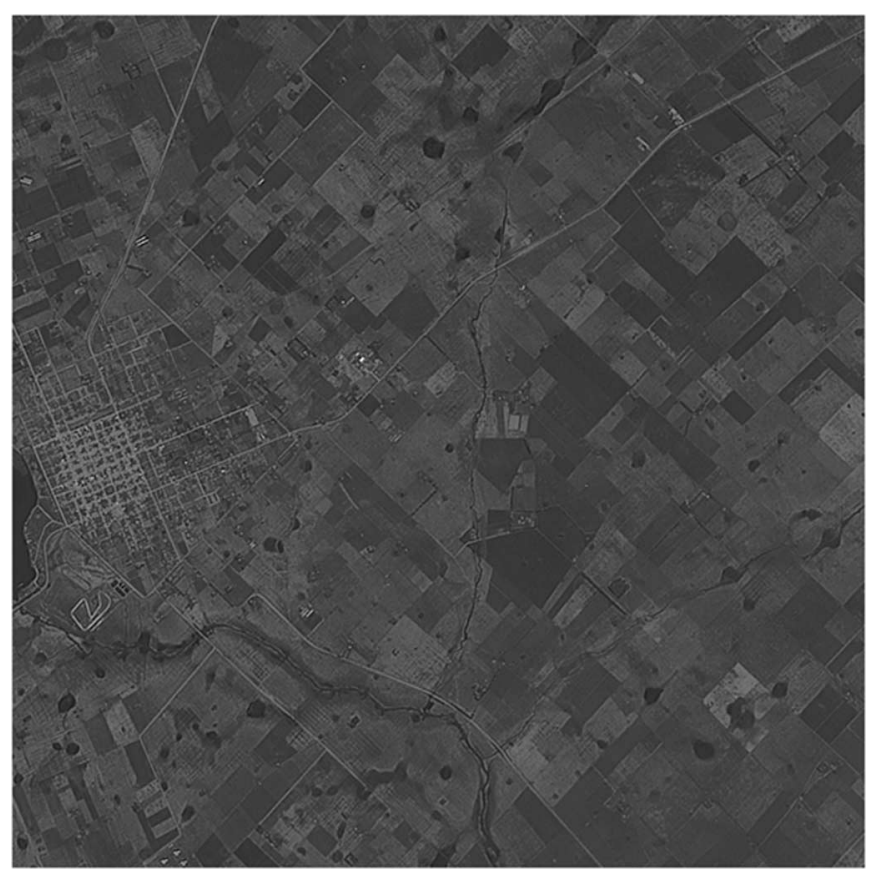

Fig. 7. High-resolution (10 m) panchromatic image of an Argentinean scene obtained by the SPOT-3 satellite.

$G=\left(B_{3}+B_{4}\right) / 2$, and $B=\left(B_{1}+B_{2}\right) / 2$. As in the previous pair, to compare the resolution with respect to the highresolution panchromatic image in the best way, we also transformed this image to panchromatic using the intensity component of the image as the mean of the three (i.e., $R, G$, and $B$ ) bands. Fig. 8 shows the low-resolution image of the pair once transformed to panchromatic. In Figs. 7 and 8, it is easy to see that the SPOT-3 and Landsat-5 images were taken at different epochs as is usual when working with images from different satellites. Note, for example, the aspect of the bed of

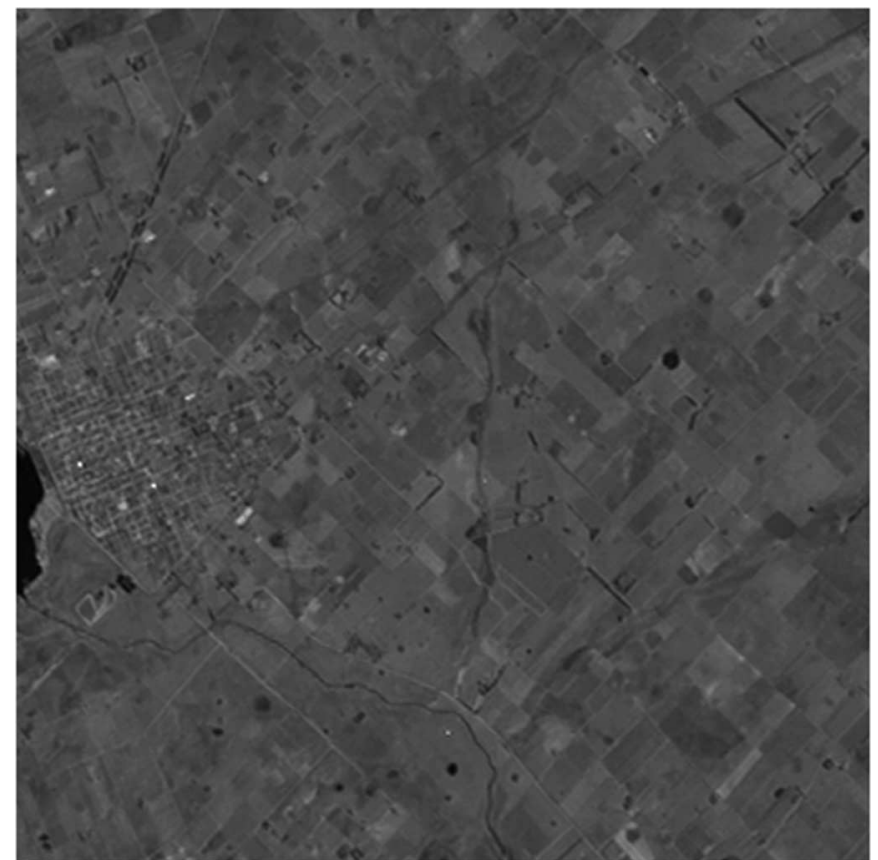

Fig. 8. Low-resolution $(30 \mathrm{~m})$ multispectral image of the same area as Fig. 7 obtained by the Landsat-5 satellite. Image was transformed to intensity levels. This image and the image in Fig. 7 were obtained during different epochs of the year.

the river, the water ponds (black rounded areas in the Landsat-5 image), or the crop fields, which, in the SPOT image, are clearly different from their appearance in the Landsat-5 image. In addition, there are several features in the SPOT picture that were not present when the Landsat-5 image was taken.

The registering between the two images of each pair was carried out, as stated above, resampling the low-resolution image using control points and a bicubic polynomial fit. Furthermore, to compensate the spectral differences between the images, we performed histogram matching between the two images of each pair using the histogram of the low-resolution image as reference to match the histogram of the high-resolution one.

As in the simulated data examples, we followed the abovedescribed methodology to compute the correlation between each image of the series obtained by wavelet decomposition of the high-resolution image (i.e., panchromatic) and the corresponding low-resolution images (i.e., color bands and intensity of the multispectral image), and fitted a cubic spline to each set of correlations. Figs. 9 and 10 show the obtained curves. Note that the B-band is not displayed in Fig. 10 because it is already displayed in Fig. 1.

Again, all curves present a maximum. The maximum correlation reached in both examples is, however, different. In the first example, the maximum correlation between the highresolution panchromatic image, and the intensity of the lowresolution multispectral image is approximately 0.59 , whereas in the second example it is approximately 0.24 ; a similar effect is observed for the spectral bands. This is a logical consequence of the different types of images involved in the presented examples. In the first example, both images come from the same satellite under the same conditions, whereas in the second example they come, as commented above, from different satellites under very different conditions. The abscissa of the maxima of 


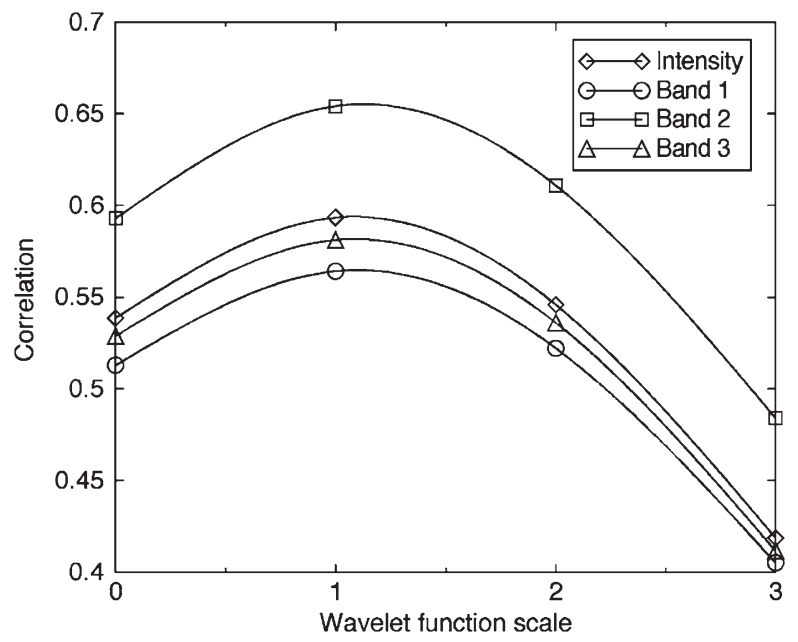

Fig. 9. Correlation curves between the high-resolution panchromatic image displayed in Fig. 5 and the intensity and spectral bands of the low-resolution multispectral image displayed in Fig. 6 as a function of the scale of the wavelet function.

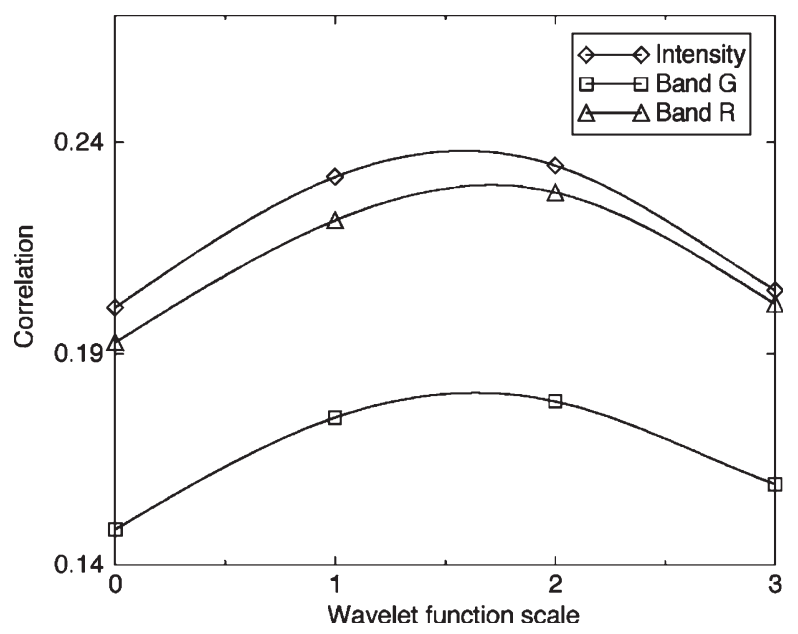

Fig. 10. Correlation curves between the high-resolution panchromatic image displayed in Fig. 7 and the intensity and spectral bands of the low-resolution multispectral image displayed in Fig. 8 as a function of the scale of the wavelet function.

the correlation curves of both examples and the computed nominal relative resolution values are summarized in Table III.

Considering first the relative resolution between the panchromatic high-resolution image and the intensity component of the low-resolution corresponding one, we observe that the maximum correlation in the first example corresponds to a scale of 1.083 , whereas the maximum in the second example is at a scale of 1.585. Applying (2), we computed the relative resolution of each pair as 2.12 and 3.00, respectively.

The computed relative resolution of the first example (i.e., 2.12 ) is close but slightly higher than the nominal resolution, which is 2. This difference can be due to several effects. Because both images of the pair were obtained under the same observing conditions by the Landsat-7 satellite, a first explanation could be that the relative performance between the panchromatic and multispectral cameras of this satellite has an actual value slightly higher than nominal. However, recent estimations of the on-orbit MTF of Landsat-7 carried out by Storey [28] and by Boggione and Fonseca [29] seem to be exactly in
TABLE III

RELATIVE RESOLUTION BETWEEN REAL IMAGES COMPUTED BY WAVELETS

\begin{tabular}{lccc}
\hline Satellites & $\begin{array}{c}\text { Maximum of } \\
\text { correlation } \\
\text { curve }(X)\end{array}$ & $\begin{array}{c}\text { Computed relative } \\
\text { resolution } \\
Y=2^{X}\end{array}$ & $\begin{array}{c}\text { Nominal } \\
\text { relative } \\
\text { resolution }\end{array}$ \\
\hline LANDSAT7-15m/ & & & \\
LANDSAT7-30m & 1.098 & 2.14 & \\
Band 1 (blue) & 1.126 & 2.18 & \\
Band 2 (green) & 1.088 & 2.13 & \\
Band 3 (red) & 1.083 & 2.12 & \\
Intensity & & & \\
SPOT3-10m/ & & & \\
LANDSAT5-30m & 1.913 & 3.77 & \\
Band B & 1.638 & 3.11 & \\
Band G & 1.712 & 3.28 & \\
Band R & 1.585 & 3.00 & \\
Intensity & & & \\
\hline
\end{tabular}

the opposite direction. From these papers, it is possible to derive that the ratio between the true absolute resolution between the panchromatic and every spectral band should be lower than 2 .

Although, as stated in Section II-C, we considered the standard low-pass filter (based on a scaling function with a $\mathbf{B}_{3}$ spline profile) to be best suited for this study, to be sure that the used filter is not disturbing the results shown in Table III for the Landsat-7 example, we carried out the same computations using the alternative "à trous" linear filter. We obtained as a result relative resolutions of $2.05,2.09,2.04$, and 2.07 for band 1 (blue), band 2 (green), band 3 (red), and intensity, respectively. These results are slightly lower than the ones displayed in Table III for the Landsat-7 example, but again, all of them are larger than 2.0. Because these results are numerically compatible with the previously obtained results and because of the reasons stated in Section II-C, we prefer to keep the original results obtained using the standard low-pass filter.

On the other hand, as stated above, the proposed method computes the relative resolution between a specific pair of images. The characteristics of the images of the pair depend on the actual size of the PSF and of the spatial frequency contents of the observed scene. Hence, in this case, the most plausible explanation of the obtained result would be the particular spatial frequency distribution present in Fig. 5. In our opinion, our results are not incompatible at all with the results in [28] and [29] because we are measuring different things. For example, in [28], Storey is measuring the true absolute resolution of Landsat-7 in both panchromatic and multispectral cameras by observing a long bridge over the surface of lake. However, we are measuring the relative resolution between a particular pair of images of a very different region (i.e., the city of Naples). Indeed, if we applied our algorithm to the particular set of images used by Storey [28], we would probably obtain that both images would have more or less the same resolution (i.e., relative resolution of approximately 1.0). This will be because the image of a bridge over an extended lake is, as a whole, an almost featureless image (except the bridge, indeed) with poor frequency content.

In the case of the second example, we obtained a relative resolution between the SPOT-3 and the intensity of the Landsat-5 images identical to the nominal one. We believe that this result could be due to chance because, even accepting that both SPOT-3 and Landsat-5 satellites could give exactly their 
nominal resolution, the different observing conditions should make at least small differences in the actual resolution of the images.

With regard to the results by spectral bands, as stated above, the proposed method works better when the SRFs of the images of the pair are closer. This is not the case when comparing a panchromatic image and the spectral bands of a multispectral one. Even so, in Table III, we can observe that the computed relative resolution for the spectral bands is quite close to both the nominal and the obtained resolutions for the intensity component. The exception is the B-band of the second example, which, as previously stated, is an extreme case because the panchromatic band of the SPOT- 3 and the blue band of the Landsat-5 satellites have very different SRFs with little overlapping.

From Figs. 9 and 10 and Table III, we can conclude that the proposed method shows good performance when estimating the relative resolution between remotely sensed images not only under ideal conditions but also under quite different circumstances as different satellites, different epochs of the year, and different types of images (panchromatic and multispectral) as in the second example.

The good performance of the method to cope with quite different types of images can also be corroborated by the successful application that we have carried out, in parallel to this paper, to compute the relative resolution between two very different types of medical images coming from positron emission tomography and magnetic resonance imaging [30].

\section{Spatial Absolute Calibrator}

The method developed in the preceding sections allows to estimate the relative resolution between two images, but we cannot compute their absolute resolution. As stated above, the actual spatial resolution of an image is difficult to know precisely, and the best way to estimate it is to know the size of a set of features appearing in the image to calibrate its absolute spatial resolution.

In this section, we describe a spatial calibrator that we have constructed to help compute the absolute resolution of a single remotely sensed image. The calibrator, which is painted on the terrace of the Department of Physics, University of Barcelona, consists of two series of parallel fringes of decreasing width and a series of triangles with a common vertex. One of the series of parallel fringes is in the north-south $(\mathrm{N}-\mathrm{S})$ direction and the other in the east-west (E-W) direction, so we can calibrate the resolution in both directions. The fringes are painted alternately in white and red color. We use the red color in place of black to facilitate the use of the calibrator when using multispectral observations. The total size of the calibrator is $18 \times 6 \mathrm{~m}$ divided in three squares of $6 \times 6 \mathrm{~m}$ (one for each group of fringes and one for the triangles). The width of the fringes are 100, 90, 80, $70,60,50,40,30$, and $20 \mathrm{~cm}$ and six fringes of $10 \mathrm{~cm}$. Each triangle is a rectangle triangle of $300-\mathrm{cm}$ basis. The size of the whole calibrator and the width of the fringes were chosen to be useful for both observations from airborne sensors and for high-resolution satellites such as SPOT-5, IKONOS, Quickbird, and other future platforms.

Fig. 11 shows an outline of the calibrator, and Fig. 12 shows a real observation of it taken from an airborne panchromatic camera. The calibrator is located at World Geodetic System

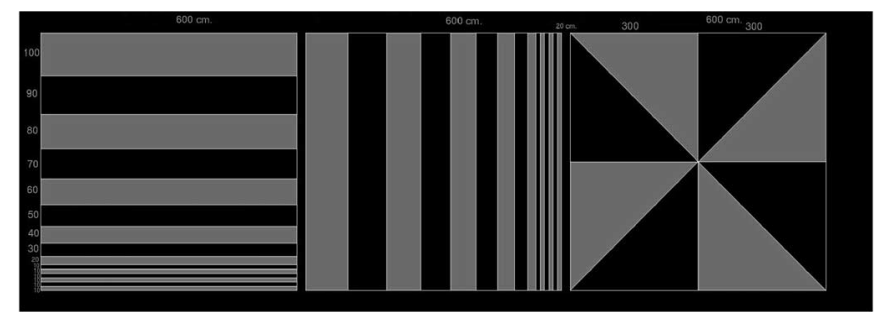

Fig. 11. Outline of the calibrator designed to estimate the absolute resolution of remotely sensed images.

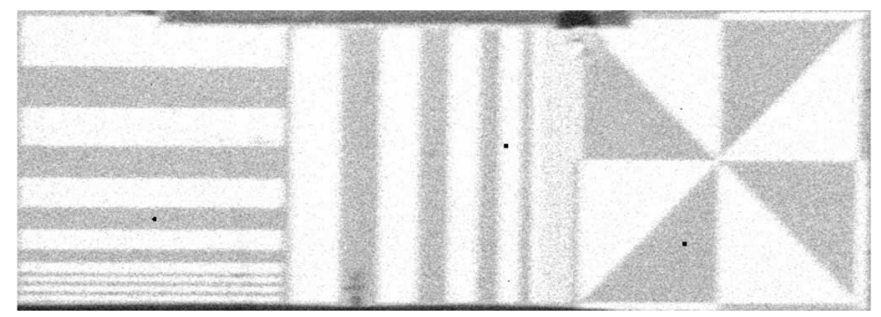

Fig. 12. Real observation of the calibrator obtained from an airborne panchromatic camera. The calibrator is located at WGS 84 coordinates: $\lambda=274.1 \mathrm{E}$; $\phi=41234.4 \mathrm{~N} ; h=95 \mathrm{~m}$ HMSL. Note the different spatial resolutions in the $\mathrm{E}-\mathrm{W}$ and $\mathrm{N}-\mathrm{S}$ directions.

coordinates (WGS 84): $\lambda=274.1 \mathrm{E} ; \phi=41234.4 \mathrm{~N}$; $h=95 \mathrm{~m}$ HMSL.

The calibrator can be used in different ways. We use two criteria to compute the resolution.

1) Because the width of the fringes is variable, the first criterion is simply to see up to which width it is possible to solve the fringes or the additional triangles in the image. Using this criterion, we can compute resolutions between $300 \mathrm{~cm}$ (the basis of the triangles) and $10 \mathrm{~cm}$ (the minimum width of the fringes).

2) If the spatial resolution is higher than $10 \mathrm{~cm}$, i.e., even all fringes of $10 \mathrm{~cm}$ are solved in the image (presently only airborne sensors can achieve this), we apply the second criterion to estimate the resolution. This is based on the Rayleigh criterion, which establishes that two punctual sources are solved if the centers of their PSFs are separated more than the radius of the same PSF. In our case, this criterion is translated into computing the intensity profile of a line perpendicular to the fringes and to study the transition zone between two consecutive fringes. We define the resolution of the image as the distance at which the intensity of a white fringe drops a half of the intensity difference with regard to the adjacent dark fringe. This method is similar to the edge technique for measuring the on-orbit MTF [31].

Fig. 13 illustrates the second estimation criterion. Fig. 13 shows a detail of an image similar to that in Fig. 12. It shows a dark fringe between two white ones and the intensity profile along a line perpendicular to them. It is easy to see that the intensity of the white fringe drops a half of the difference between adjacent fringes at approximately 4.5 pixels. To compute the corresponding resolution, we also need the scale of the image, but because we know the true size of the fringes, computing the scale in centimeters per pixel is straightforward.

Fig. 12 also illustrates that the calibrator can be used to detect differences of resolution in different directions due to problems 


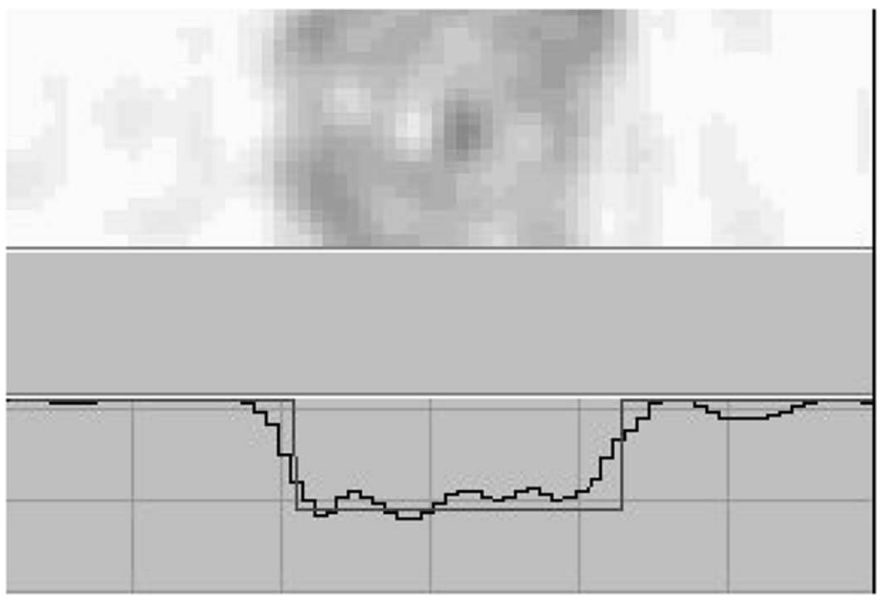

Fig. 13. Illustration of the Rayleigh criterion to compute the absolute resolution of an image. The figure shows a dark fringe of the calibrator between two white ones and the intensity profile along a line perpendicular to them.

in the optics or to errors in the motion compensator. Note that in Fig. 12 the resolution in the $\mathrm{E}-\mathrm{W}$ direction (i.e., horizontal) is clearly worse than in the $\mathrm{N}-\mathrm{S}$ direction (i.e., vertical). It can be easily seen because the $10-\mathrm{cm}$ horizontal fringes at the left are clearly visible, whereas the $10-\mathrm{cm}$ vertical fringes at the center right are not. This is probably due to a bad calibration of the motion compensator of the airborne camera that took the image. In a case like this, we can use the aforementioned first criterion to estimate the resolution in the $\mathrm{E}-\mathrm{W}$ direction and the second one for the $\mathrm{N}-\mathrm{S}$ direction. In this particular example, we obtained an approximately $10-\mathrm{cm}$ resolution in the E-W direction but less than $5-\mathrm{cm}$ resolution (applying the second criterion) in the $\mathrm{N}-\mathrm{S}$ direction.

We should note that because the calibrator is located at a fixed place, its use is, in principle, restricted to calibrate observations that include it. However, given that remote sensing satellites can observe a specified area as many times as desired, it is possible to use the calibrator to obtain the true resolution of a series of images taken under different conditions of illumination, dust, pollution, or weather. This can help to better calibrate the actual resolution of the satellite under such conditions or to calibrate a possible malfunction of the satellite. The combination of this true resolution calibration and the relative resolution computation presented above can help to better estimate the absolute resolution of any image.

\section{CONCLUSION}

The actual spatial resolution of remotely sensed images is a key parameter but is difficult to know with high accuracy. The aim of this paper was to establish a method for estimating the relative and absolute resolution of such images.

The proposed relative method, which is useful for the determination of the relative resolution between a specific pair of images of the same area, is based on the mathematical properties of wavelet decomposition, in particular in the dyadic properties of the "à trous" algorithm of the wavelet decomposition. The proposed method obtains the relative resolution from the computation of the maximum correlation between a series of images of decreasing resolution of the high-resolution image and the lower resolution image of the pair. The method works well with panchromatic and multispectral images.
To show the robustness of the method, we applied it to simulated data and to two very different pairs of real images. One of them was a pair of real images obtained by the panchromatic and multispectral sensors of the Landsat-7 satellite under identical conditions. The other pair of real data was composed by a panchromatic image obtained by the SPOT- 3 satellite and a multispectral image obtained at different epochs of the year by the Landsat- 5 satellite, thus under very different conditions. In both cases, we computed the relative resolution between the images of the pair. Thus, we can conclude that the proposed method is robust in estimating the relative resolution between remotely sensed images not only under ideal conditions but also for different satellites and under different circumstances such as epoch of the year and spectral characteristics of the images.

Because the proposed method can only estimate the relative resolution of a specific pair of images, in Section IV, we presented the design, characteristics, and use of a spatial calibrator we constructed on the terrace of our department. Its design helps to compute the absolute resolution of an image in both $\mathrm{N}-\mathrm{S}$ and $\mathrm{E}-\mathrm{W}$ directions, allowing to detect possible differences in resolution with direction. Its size makes it useful for both airborne sensors and high-resolution satellites. The calibrator can be used to better establish the actual resolution of a satellite under different conditions of weather, illumination, and pollution, and to detect possible malfunctions of the satellite.

To use the calibrator, we presented a direct estimation method and another method based on the Rayleigh criterion, which allows to compute absolute resolutions from $3 \mathrm{~m}$ to a few centimeters. We presented a real example of its use that allowed us to detect appreciable differences in resolution in the $\mathrm{E}-\mathrm{W}$ and $\mathrm{N}-\mathrm{S}$ directions.

Finally, the combination of the relative and absolute methods presented in this paper can help to estimate the absolute resolution of any image.

\section{ACKNOWLEDGMENT}

The authors would like to thank the three anonymous reviewers for their detailed and useful comments that helped improve the contents and references of this paper.

\section{REFERENCES}

[1] D. L. Fried, "Optical resolution through a randomly inhomogeneous medium for very long and very short exposures," J. Opt. Soc. Amer. vol. 56 , no. 10 , pp. $1372-1379$, Oct. 1966.

[2] _ "Limiting resolution looking down through the atmosphere," J. Opt. Soc. Amer, vol. 56, no. 10, pp. 1380-1384, Oct. 1966.

[3] Y. J. Kaufman, "Atmospheric effects on spatial resolution of surface imagery," Appl. Opt., vol. 23, no. 22, pp. 4164-4172, Nov. 1984.

[4] N. S. Kopeika, I. Dror, and D. Sadot, "Causes of atmospheric blur: Comment on atmospheric scattering effect on spatial resolution of imaging systems," J. Opt. Soc. Amer. A, Opt. Image Sci., vol. 15, no. 12, pp. 3097-3106, Dec. 1998.

[5] S. C. Park, M. K. Park, and M. G. Kang, "superresolution image reconstruction: A technical overview," IEEE Signal Process. Mag., vol. 20, no. 3, pp. 21-36, May 2003.

[6] X. Otazu, M. González-Audícana, O. Fors, and J. Núñez, "Introduction of sensor spectral response into image fusion methods. Application to wavelet-based methods," IEEE Trans. Geosci. Remote Sens., vol. 43, no. 10, pp. 2376-2385, Oct. 2005.

[7] Y. Meyer, Wavelets. Algorithms and Applications. Philadelphia, PA: SIAM, 1993.

[8] R. K. Young, Wavelet Theory and Its Applications. Boston, MA: Kluwer, 1993. 
[9] I. Daubechies, Ten Lectures on Wavelets. Philadelphia, PA: SIAM Press, 1992.

[10] C. K. Chui, An Introduction to Wavelets. Boston, MA: Academic, 1992.

[11] G. Kaiser, A Friendly Guide to Wavelets. Boston, MA: Birkhauser, 1994.

[12] M. Vetterli and J. Kovacevic, Wavelets and Subband Coding. Englewood Cliffs, NJ: Prentice-Hall, 1995.

[13] J. L. Starck and E. Pantin, "Multiscale maximum entropy image restoration," Vistas Astron., vol. 40, no. 4, pp. 563-569, 1996.

[14] F. Rué and A. Bijaoui, "A multiscale vision model applied to astronomical images," Vistas Astron., vol. 40, no. 4, pp. 495-502, 1996.

[15] S. Mallat, "A theory for multiresolution signal: The wavelet representation," IEEE Trans. Pattern Anal. Mach. Intell., vol. 11, no. 7, pp. 674-693, Jul. 1989.

[16] — - A Wavelet Tour of Signal Processing, 2nd ed. San Diego, CA: Academic, 1998.

[17] M. Datcu, D. Luca, and K. Seidel, "Multiresolution analysis of SAR images," in Proc. EUSAR, Konigswinter, Germany, 1996, pp. 375-378.

[18] D. A. Yocky, "Image merging and data fusion by means of the discrete two-dimensional wavelet transform," J. Opt. Soc. Amer. A, Opt. Image Sci., vol. 12, no. 9, pp. 1834-1841, Sep. 1995.

[19] T. Ranchin, L. Wald, and M. Mangolini, "The ARSIS method: A general solution for improving spatial resolution of images by the means of sensor fusion," in Proc. Int. Conf.: Fusion Earth Data, T. Ranchin and L. Wald, Eds. Cannes, France, 1996, pp. 53-58.

[20] L. Wald, T. Ranchin, and M. Mangolini, "Fusion of satellite images of different spatial resolution: Assessing the quality of resulting images," Photogramm. Eng. Remote Sens., vol. 63, no. 6, pp. 691-699, 1997.

[21] J. Núñez, X. Otazu, O. Fors, V. Palà, and R. Arbiol, "Multiresolutionbased image fusion with additive wavelet decomposition," IEEE Trans. Geosci. Remote Sens., vol. 37, no. 3, pp. 1204-1211, May 1999.

[22] J. Núñez, X. Otazu, O. Fors, A. Prades, V. Palà, and R. Arbiol, "Image fusion using additive multiresolution wavelet decomposition. Applications to SPOT + Landsat images," J. Opt. Soc. Amer. A, Opt. Image Sci., vol. 16, no. 3, pp. 467-474, Mar. 1999.

[23] M. González-Audícana, X. Otazu, O. Fors, and A. Seco, "Comparison between the Mallat's and the à trous discrete wavelet transform based algorithms for the fusion of multispectral and panchromatic images," Int. J. Remote Sens., vol. 26, no. 3, pp. 597-616, Feb. 2005.

[24] J. L. Starck and F. Murtagh, "Image restoration with noise suppression using the wavelet transform," Astron. Astrophys., vol. 228, no. 1, pp. 342-350, Aug. 1994.

[25] M. Holschneider and P. Tchamitchian, Les Ondelettes En 1989, P. G. Lemarié, Ed. Paris, France: Springer-Verlag, 1990, p. 102.

[26] M. J. Shensa, "The discrete wavelet transform: Wedding the "à trous" and Mallat algorithms," IEEE Trans. Signal Process., vol. 40, no. 10, pp. 2464-2482, Oct. 1992.

[27] T. Ranchin, B. Aiazzi, L. Alparone, S. Baronti, and L. Wald, "Image fusion-The ARSIS concept and some successful implementations schemes," ISPRS J. Photogramm. Remote Sens., vol. 58, no. 1/2, pp. 4-18, Jun. 2003.

[28] J. C. Storey, "Landsat 7 on-orbit modulation transfer function estimation," Proc. SPIE, vol. 4540, pp. 50-61, 2001.

[29] G. A. Boggione and L. M. G. Fonseca, "Restoration of Landsat-7 images," in Proc. 30th Symp. Remote Sens. Environ.: Inf. Risk Manage. Sustain. Develop., Honolulu, HI, Nov. 10-14, 2003, p. PS-I.4. CD-ROM.

[30] J. Núñez, X. Otazu, and M. T. Merino, "A multiresolution-based method for the determination of the relative resolution between images. First application to remote sensing and medical images," Int. J. Imaging Syst. Technol., vol. 15, no. 5, pp. 225-235, 2005.

[31] B. Tatain, "Method for obtaining the transfer function from the edge response function," J. Opt. Soc. Amer. A, Opt. Image Sci., vol. 55, no. 8, pp. 1014-1019, Aug. 1965.

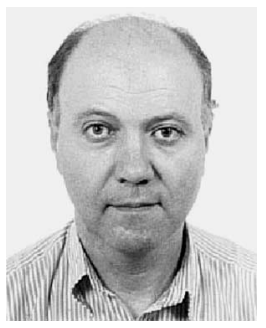

Jorge Núñez received the B.A. and Ph.D. degrees in physics from the Universitat de Barcelona, Barcelona, Spain, in 1975 and 1981, respectively.

Since 1984, he has been a Professor with the Department of Astronomy, Universitat de Barcelona. In 1998, he was elected member of the Academy of Sciences and Arts of Barcelona. He is currently conducting research on digital image processing including image reconstruction and restoration using Bayesian techniques and data fusion with applications in astronomy, medicine, and remote sensing.

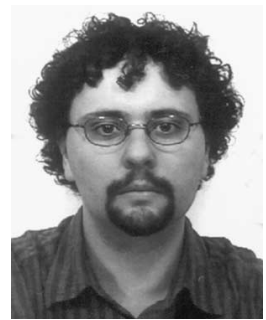

Octavi Fors received the B.A. degree in physics from the Universitat de Barcelona, Barcelona, Spain, in 1996, the M.S. degree in remote sensing from the Institut d'Estudis Espacials de Catalunya, Barcelona, in 1999, and the Ph.D. degree in physics from the Universitat de Barcelona, in 2006.

Since 1996, he has been working on image processing techniques in the fields of astronomy and remote sensing. His research interests include image deconvolution, image fusion, and multiresolutionbased disparity analysis.

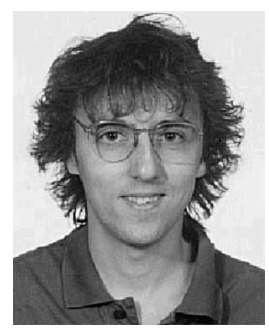

Xavier Otazu received the B.A. degree in physics from the Universitat de Barcelona, Barcelona, Spain, in 1994, the M.S. degree in remote sensing from the Institut d'Estudis Espacials de Catalunya, Barcelona, in 1999, and the Ph.D. degree in physics from the Universitat de Barcelona, in 2001.

He was with the R\&D Remote Sensing Group, Institut Cartogràfic de Catalunya, Spain. He is currently a Ramón y Cajal Researcher with the Centre de Visió per Computador, Universitat Autónoma de Barcelona, Bellaterra, Spain. Since 1994, he has been involved in the applications of wavelets into astronomical and remote sensing data processing. His research interests include image deconvolution, image fusion, and description of the perceptual processes of the human visual system.

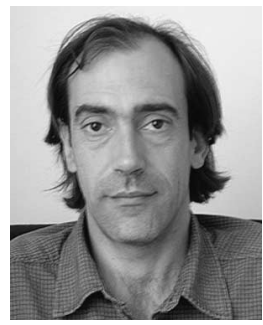

Vicenç Palà received the B.Eng. degree in computer science from the Universitat Politècnica de Catalunya (UPC), Barcelona, Spain, in 1984.

From 1982 to 1986, he was a Researcher with the UPC Computing Center, where he developed digital processing tools in the remote sensing domain. Since 1987, he has been the Head of the Image Processing and Systems Development, Institut Cartogràfic de Catalunya, Spain, where he has been involved in projects related to image processing and geocoding.

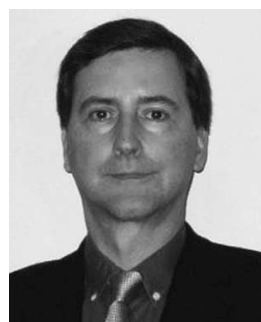

Román Arbiol received the B.S. degree in physics from the Universitat de Barcelona, Barcelona, Spain, in 1976, and the B.S. degree in computer science from the Polytechnic University of Catalonia, Barcelona, in 1984.

Since 1980, he has been involved in developing image processing algorithms to extract information from Earth observation satellite images. Since 1983, he has been the Head of the Image Processing and Remote Sensing Department, Cartographic Institute of Catalonia. His research interests include image restoration, image classification, and geometrical models.

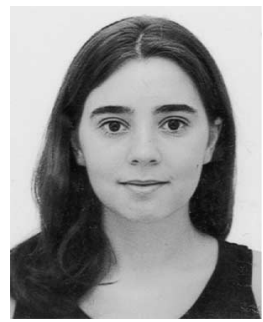

Maria Teresa Merino received the B.S. degree in physics from the Universitat de Barcelona (UB), Barcelona, Spain, in 2002, and the M.Sc. degree in remote sensing from the Institut d'Estudis Espacials de Catalunya, Barcelona, in 2004. She is currently working toward the Ph.D. degree in physics at UB.

Since 2004, she has been working on image processing techniques in the fields of astronomy and remote sensing. Her research interests include superresolution and image deconvolution. 